\title{
MECHANICAL PROPERTIES OF TI-6AL-4V SAMPLES PRODUCED WITH ADDITIVE TECHNOLOGY
}

\author{
Zoltan Keresztes, $^{1, \mathrm{a}}$, David Pammer ${ }^{1, \mathrm{~b}, *}$
}

1 Budapest University of Technology and Ecomonics, Department of Materials Science and Technology, Bertalan Lajos str. 7, 1111, Budapest, Hungary. ${ }^{\mathrm{a} E} \mathrm{E}-\mathrm{mail}$ : kzoltan@eik.bme.hu, b, $\quad$ corresponding author E-mail: pammer@eik.bme.hu

\section{Introduction}

Rapid Prototyping is a term used to describe fabrication processes responsible for creating representations and models during the development of a project. In industrial context, the expression usually refers to technologies that are able to build prototypes from digital data. Nowadays the name of these processes are changed to Additive Manufacturing (AM), due to the most basic features of the technology becomes the fabrication of parts using an additive approach [1]. Synonyms: additive processes, additive fabrication, etc. [2].

The process starts with a computer-aided design software, which sends the information to a professional-grade industrial machine. This machine builds the final 3D objects layer-by-layer from different raw material based on the descriptions sent by the software [3].

Our main aim was to compare the mechanical properties and the microstructures of Grade $5 \mathrm{Ti}$ $6 \mathrm{Al}-4 \mathrm{~V}$ samples produced by traditional processes and additive manufacturing. This work represents several tests, such as tensile, bending, hardness and metallography. All samples and tests fulfill the available ASTM and ISO standards.

\section{Materials and methods}

During the tests Ti-6Al-4V samples were used, with the following chemical composition (Tab.1.).

\begin{tabular}{|c|c|}
\hline Component & Percentage (\%) \\
\hline Titanium (Ti) & $88.1-91$ \\
\hline Aluminum (Al) & $5.5-6.5$ \\
\hline Vanadium (V) & $3.5-4.5$ \\
\hline Iron (Fe) & $<0.25$ \\
\hline Oxygen (O) & $<0.13$ \\
\hline Carbon (C) & $<0.08$ \\
\hline Nitrogen (N) & $<0.03$ \\
\hline Hydrogen (H) & $<0.125$ \\
\hline
\end{tabular}

Tab.1. Chemical composition of Ti-6Al-4V produced by traditional process

AM has many types, such the main processes as: VAT photopolymerisation; Material Jetting; Binder Jetting; Material Extrusion; Powder Bed Fusion; Sheet Lamination; Direct Energy Deposition.

The samples were produced by a 3D printing technology of Powder Bed Fusion called Direct Metal Laser Sintering (DMLS). During the production with EOS M 280 machine the model is built by very thin layers and fused raw powder. The schematic illustration of the 3D printing technology is showed below (Fig.1.) [4].

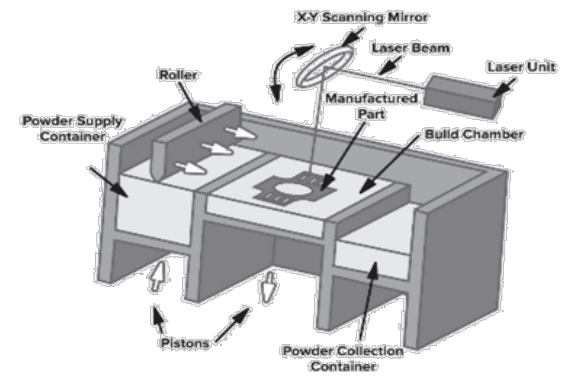

Fig.1. Schematic illustration of DMLS technology

\section{Mechanical properties and tests}

The tensile specimens were built following ASTM E8M:11. The specimens total length was $50 \mathrm{~mm}$. The bending specimens were built with a total length of $50 \mathrm{~mm}$ also and a cross section of $4 \mathrm{~mm} \times 4 \mathrm{~mm}$, where the geometry do not influence the manufacturing direction, because there symmetricity. The manufacturing direction of the tensile specimens are shown on Fig.2.

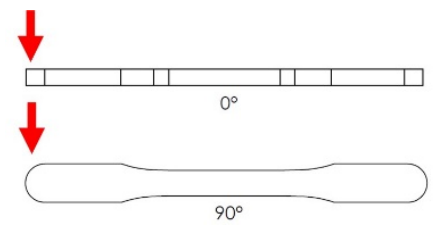

Fig.2. Tensile specimens with themanufacturing direction 


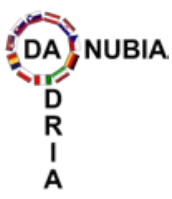

34th Danubia-Adria Symposium on Advances in Experimental Mechanics

University of Trieste, Italy, 2017
Tensile and bending (TPB) test was performed with Instron 5965 tensile test machine, with 6-6 specimens (Fig.3. and Fig.4.). 3 with 0 deg and 3 with 90 deg manufacturing direction.

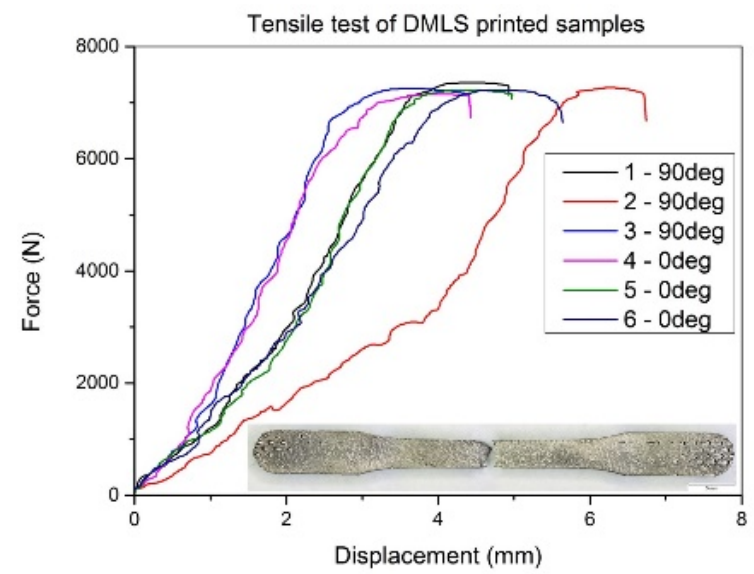

Fig.3. Tensile test of DMLS produced Ti-6Al-4V parts

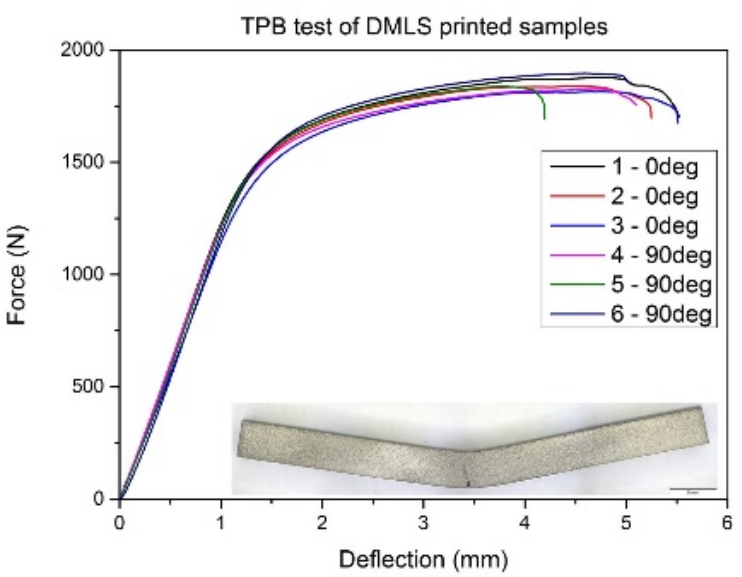

Fig.4. Bending test of DMLS produced Ti-6Al-4V parts

The stress-strain values were calculated after the measurement. Tensile strength: $1134 \pm 10.67 \mathrm{MPa}$, and flexural resistance: $1957.5 \pm 31.05 \mathrm{MPa}$.

\section{Metallography}

For a metallography study first of all the samples must be fixed in resin to make the grinding, polishing and etching effective. For etching, we used Keller's reagent ( $190 \mathrm{ml} \mathrm{H}_{2} \mathrm{O}$, 5 $\mathrm{ml} \mathrm{HNO}_{3}, 3 \mathrm{ml} \mathrm{HCl}, 2 \mathrm{ml} \mathrm{HF}$ ), which is optimal for titan alloys. Fig. 5. shows the microstructure of Ti-6Al-4V produced traditionally and Fig.3. represents the microstructure of additive manufactured Ti-6Al-4V sample etched with Keller's reagent.

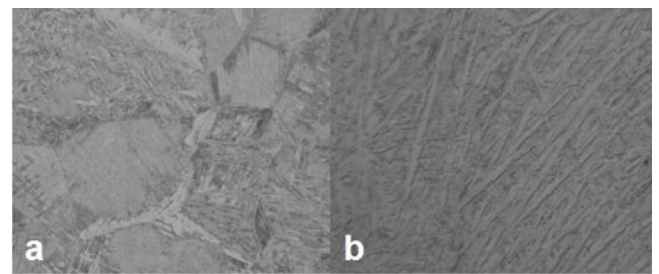

Fig.5. a) Microstructure of traditionally produced Ti6Al-4V parts; b) Microstructure of DMLS produced Ti$6 \mathrm{Al}-4 \mathrm{~V}$ parts

For more precise information the microstructures were investigated by Inspect S50 $30 \mathrm{kV}$ SEM. Two different areas were examined. Firstly, the outer part of the sample was tested and secondly the inner area was investigated (Fig.6.). We inspected the chemical composition, were $\mathrm{Ti}=86.86 \%$, $\mathrm{Al}=10.58 \%$ and $\mathrm{V}=2.56 \%$.

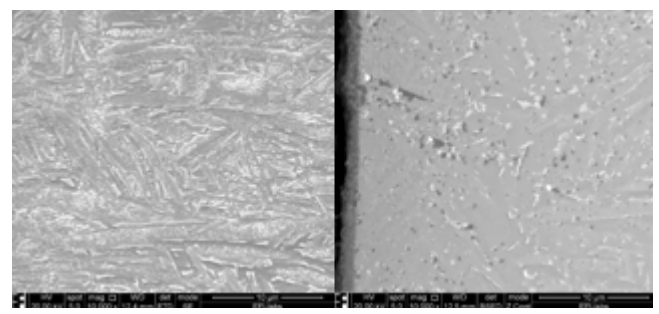

Fig.6. SEM pictures of the DMLS produced sample, outer part (left), inside part (right)

\section{Remarks}

Considering the average tension results for Ti$6 \mathrm{Al}-4 \mathrm{~V}$, which varies between $900 \mathrm{MPa}$ and 1200 MPa, the DMLS samples have values over 1100 $\mathrm{MPa}$, independent of the manufacturing direction ( 0 or $90 \mathrm{deg}$ ), and the near similar microstructure and chemical composition causes that the DMLS produced parts can be used in the same application fields as the traditional manufacturing method.

\section{Acknowledgements}

The present work was funded by the National Research, Development and Innovation Fund, connecting to the NVKP_16-1-2016-0022 project.

\section{References}

[1] Ian Gibson, David Rosen, Brent Stucker, Additive Manufacturing Technologies. 2. ed. New York, Springer, 2009.

[2] William E. Frazier, Metal Additive Manufacturing: Journal Of Materials Engineering And Performance, 2014

[3] Sharon L.N. Ford, Metal Additive Manufacturing Technology: Potential 

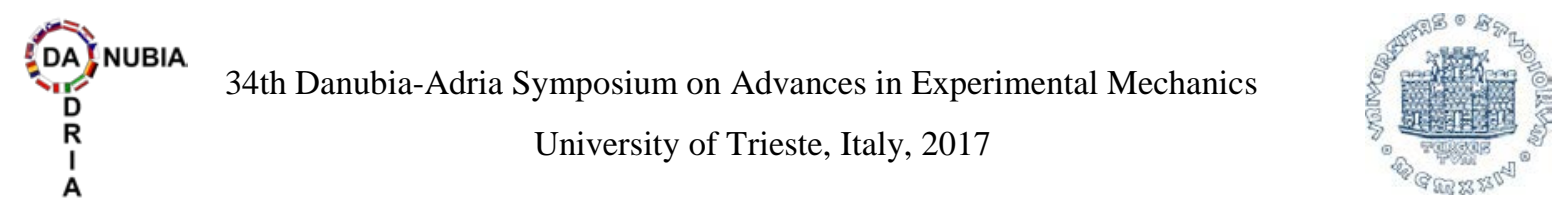

Implications for U.S. Manufacturing Competitiveness, Journal Of International Commerce And Economics, 2014

[4] Loughborough University, The 7 Categories of Additive Manufacturing, http://www.lboro.ac.uk/research/amrg/about/t he7categoriesofadditivemanufacturing, 2016 